\title{
Experimental Study of $\mathrm{O}_{2}+\mathrm{CO}=\mathrm{CO}_{2}+\mathrm{O}$ Reaction Kinetics behind Shock Waves at Different Pressures
}

\author{
A.V. Drakon ${ }^{1}$, A.V. Emelianov ${ }^{1}$, A.V. Eremin ${ }^{1}$, P.I. Yatsenko ${ }^{1,2}$ \\ ${ }^{1}$ Joint Institute for High Temperatures of the Russian Academy of Sciences, \\ Moscow, 125412, Russia \\ ${ }^{2}$ Bauman Moscow State Technical University, \\ Moscow, 105005, Russia \\ pavelyatcenko@yandex.ru
}

\begin{abstract}
The time profile measurements of the $\mathrm{O}$ atoms concentration formed during the interaction of molecular oxygen with carbon monoxide were performed behind the reflected shock waves using method of atomic resonance absorption spectroscopy (ARAS). The experiments were carried out in mixture of $0.1 \% \mathrm{O}_{2}+0.1 \% \mathrm{CO}+\mathrm{Ar}$ at temperature of $1200 \div 2200 \mathrm{~K}$ and pressures of 2.5 bar and 16.5 bar. Based on the results of the experimental data, the rate constants $k_{f}$ of forward reaction $\mathrm{O}_{2}+\mathrm{CO}+\mathrm{Ar} \rightarrow \mathrm{CO}_{2}+\mathrm{O}+\mathrm{Ar}$ were determined at different pressures:

$$
\begin{gathered}
k_{f H}=3.17 \times 10^{11} \exp \left(-\frac{167 \mathrm{~kJ}}{R T}\right), \mathrm{cm}^{3} \cdot \mathrm{mole}^{-1} \cdot \mathrm{s}^{-1}(P=16.5 \mathrm{bar}) \\
k_{f L}=1.08 \times 10^{13} \exp \left(-\frac{167 \mathrm{~kJ}}{R T}\right), \mathrm{cm}^{3} \cdot \mathrm{mole}^{-1} \cdot \mathrm{s}^{-1}(P=2.5 \mathrm{bar})
\end{gathered}
$$

The performed calculations indicate the effect of the gas mixture pressure on the reaction rate constant can be explained within the activated complex theory (TAC) by the different time relationship between gas-kinetic collisions and the characteristic $\mathrm{O}_{2}+\mathrm{CO}$ interaction time. On the basis of this theory, model calculations of the rate constants were carried out, and the lifetime of the activated complex $\left(\mathrm{O}_{2}: \mathrm{CO}\right)$, equal to $0.5 \pm 0.3 \mathrm{~ns}$, was determined.
\end{abstract}

Keywords: combustion, chemical kinetics, reaction rate constants, shock tube, atomic resonance absorption spectroscopy (ARAS). 


\title{
Экспериментальное исследование кинетики реакции $\mathrm{O}_{2}+\mathrm{CO}=\mathrm{CO}_{2}+\mathrm{O}$ за ударными волнами при различном давлении
}

\author{
А.В. Дракон ${ }^{1}$, А.В. Емельянов ${ }^{1}$, А.В. Еремин ${ }^{1}$, П.И. Яценко ${ }^{1,2}$ \\ ${ }^{1}$ Объединенный институт высоких температур Российской академии наук, \\ Россия, Москва, 125412, ул. Ижорская, д.13 \\ ${ }^{2}$ Московский государственный технический университет имени Н.Э. Баумана, \\ Россия, Москва, 105005, 2-я Бауманская ул., 5 \\ pavelyatcenko@yandex.ru
}

\begin{abstract}
Аннотация
В работе методом атомной резонансной абсорбционной спектроскопии (АРАС) проведены измерения временных профилей концентрации атомов $\mathrm{O}$, образующихся при взаимодействии молекулярного кислорода с монооксидом углерода за отраженными ударными волнами. Эксперименты проведены в смеси $0.1 \% \mathrm{O}_{2}+0.1 \% \mathrm{CO}+\mathrm{Ar}$ при температуре $1200 \div 2200 \mathrm{~K}$ и давлениях в 2.5 бар и 16.5 бар. По результатам экспериментальных данных определены константы скорости прямой $k_{f}$ реакции $\mathrm{O}_{2}+\mathrm{CO}+\mathrm{Ar} \rightarrow \mathrm{CO}_{2}+\mathrm{O}+\mathrm{Ar}$ при различном давлении

$$
\begin{gathered}
k_{f H}=3.17 \times 10^{11} \exp \left(-\frac{167 k J}{R T}\right), \mathrm{cm}^{3} \cdot \text { моль }^{-1} \cdot \mathrm{c}^{-1}(P=16.5 \text { бар }) \\
k_{f L}=1.08 \times 10^{13} \exp \left(-\frac{167 \mathrm{~kJ}}{R T}\right), \mathrm{cm}^{3} \cdot \text { моль }^{-1} \cdot \mathrm{c}^{-1}(P=2.5 \text { бар })
\end{gathered}
$$

Проведенные расчеты показывают, что влияние давления газовой смеси на величину константы скорости реакции может объясняться различным временным соотношением между газокинетическими соударениями и характерным временем взаимодействия $\mathrm{O}_{2}+\mathrm{CO}$ в рамках теории активированного комплекса (ТАК). На основе этой теории проведены модельные расчеты констант скоростей, а также определено время жизни активированного комплекса $\left(\mathrm{O}_{2}: \mathrm{CO}\right)$, равное $0.5 \pm 0.3 \mathrm{Hc}$.
\end{abstract}

Ключевые слова: горение, химическая кинетика, константы скорости реакции, ударная труба, атомно-резонансная абсорбционная спектроскопия.

\section{1. Введение}

Обзор реакций, играющих важную роль в развитии воспламенения и детонации различных углеводородных, а также перспективных синтезгазо-воздушных топлив показал, что реакция $\mathrm{CO}+\mathrm{O}_{2}=\mathrm{CO}_{2}+\mathrm{O}$ вносит существенный вклад в общую картину цепного механизма горения многих веществ. Еще одной причиной интереса к данной реакции является не часто встречаемое сочетание энергетических параметров. Экзотермический процесс взаимодействия моноксида углерода с кислородом является высокобарьерным. По подсчетам различных авторов [1-11] энергия активации $E$ варьируется в пределах от 100 до 270 кДж/моль, а тепловой эффект $Q$ реакции в условиях эксперимента является весьма низким и составляет порядка -52 кДж/моль [12]. Такое соотношение параметров делает реакцию $\mathrm{CO}+\mathrm{O}_{2}$ невыгодной с энергетической точки зрения. Однако образующиеся в ходе этой реакции радикалы могут вносить свой значительный вклад в развитие цепного механизма воспламенения. Поэто- 
му, детальный анализ кинетики взаимодействия $\mathrm{CO}$ с $\mathrm{O}_{2}$, надежный контроль количества реагентов и поиск оптимального соотношения состава компонентов может позволить улучшить характеристики энергетических установок, использующих синтезгаз.

Вследствие чрезвычайной практической важности интерес к кинетике этой реакции проявляется достаточно давно и не утихает до сих пор. С 50-х годов двадцатого века, когда значительно улучшились методы оптической диагностики, вопросами ее изучения занялись на ударно-трубных установках [1]. К настоящему времени известно более 20 экспериментальных и теоретических работ по кинетике реакции окиси углерода с молекулярным кислородом. Наиболее значимые работы представлены в таблице 1. Экспериментальные подходы в этих работах включали как анализ времен задержек воспламенения в горючих водородкислородных смесях, содержащих примесь СО с последующим применением расчетов, так и прямые абсорбционные измерения концентрации атомарного кислорода в сильноразбавленных смесях $\mathrm{CO}+\mathrm{O}_{2}$. Отдельного внимания заслуживает работа [2], в которой значение константы реакции было извлечено косвенным образом в ходе анализа полученных методом AРАC на длине волны резонансной линии атома Н (Лайман-Альфа) 121.6 нм временных профилей концентрации атомарного водорода при развитии воспламенения в смеси $0.2 \% \mathrm{H}_{2}+1 \% \mathrm{O}_{2}+1 \% \mathrm{CO}+\mathrm{Ar}$. Это позволило получить данные в диапазоне низких температур $(1000 \div 1200 \mathrm{~K})$, который остался неисследованным в более ранних работах.

Таблий 1

Литературные данные по значениям скорости реакции $\mathrm{CO}+\mathrm{O}_{2}=\mathrm{CO}_{2}+\mathrm{O}$

\begin{tabular}{|c|c|c|c|c|c|}
\hline \multirow{2}{*}{ № } & \multirow{2}{*}{ Источник } & \multirow{2}{*}{$\begin{array}{c}\text { Диапазон } \\
\text { температур, K }\end{array}$} & \multirow{2}{*}{$\begin{array}{c}\text { Диапазон } \\
\text { давлений, Па }\end{array}$} & \multicolumn{2}{|c|}{$k=A \exp \left(-E_{a} / R T\right)$} \\
\hline & & & & $\begin{array}{c}A, \\
\mathrm{~cm}^{3} / \text { моль } * \mathrm{c}\end{array}$ & $\begin{array}{c}E_{a}, \\
\text { кДж/моль }\end{array}$ \\
\hline \multicolumn{6}{|c|}{ Экспериментальные исследования } \\
\hline 1 & 1957FEN [3] & $1700 \div 2000$ & $10^{5}$ & $1.20 \times 10^{12}$ & 101 \\
\hline 2 & 1965SUL [4] & $2400 \div 3000$ & $10^{5}$ & $3.50 \times 10^{12}$ & 214 \\
\hline 3 & 1968DRU [5] & $1190 \div 1850$ & $2.23 \div 4.27 \times 10^{5}$ & $2.40 \times 10^{10}$ & 127 \\
\hline 4 & 1970DEA [6] & $1750 \div 2580$ & $2 \times 10^{3}$ & $1.21 \times 10^{13}$ & 251 \\
\hline 5 & 1970DEA2 [7] & $2200 \div 2600$ & $2.2 \times 10^{3}$ & $3.49 \times 10^{12}$ & 210 \\
\hline 6 & 1971BRA [8] & $1300 \div 1900$ & $0.91 \div 1.56 \times 10^{5}$ & $1.60 \times 10^{13}$ & 171 \\
\hline 7 & 1971GAR [9] & $1400 \div 2500$ & $1.5 \div 3.0 \times 10^{4}$ & $3.10 \times 10^{11}$ & 159 \\
\hline 8 & 1974RAW [10] & $1500 \div 2500$ & $1.5 \div 3.0 \times 10^{4}$ & $1.20 \times 10^{11}$ & 146 \\
\hline 9 & 1983THI [11] & $1700 \div 3500$ & $1.8 \times 10^{5}$ & $5.06 \times 10^{13}$ & 264 \\
\hline 10 & $1991 \mathrm{KOI}[2]$ & $1000 \div 1400$ & $2.66 \times 10^{3}$ & $1.26 \times 10^{13}$ & 197 \\
\hline \multicolumn{6}{|c|}{ Обзоры } \\
\hline 11 & 1984WAR [13] & $1500 \div 3000$ & - & $2.50 \times 10^{12}$ & 200 \\
\hline 12 & 1986TSA [14] & $300 \div 2500$ & - & $2.53 \times 10^{12}$ & 200 \\
\hline 13 & 1991IBR [15] & $1700 \div 6000$ & - & $5.06 \times 10^{13}$ & 264 \\
\hline
\end{tabular}

Но, несмотря на большое количество проведенных на данный момент исследований, однозначных и всеобъемлющих данных по кинетике реакции монооксида углерода с атомар- 
ным кислородом получить не удалось. Подчас результаты противоречат друг другу, а значения константы скорости отличаются на порядки. Нерешенность задачи, наряду с ее практической значимостью, показывает, что исследование кинетики этой реакции остается весьма актуально, с научной точки зрения, и по сей день.

Основной целью данного исследования явилось получение новых уточненных экспериментальных данных по константе скорости реакции $\mathrm{CO}+\mathrm{O}_{2}$ путем применения прецизионного метода атомно-резонансной абсорбционной спектроскопии для измерения временных профилей концентрации атомарного кислорода в широком диапазоне температур, а также изучение зависимости константы скорости от величины давления.

\section{2. Эксперимент}

\section{1. Экспериментальная установка}

Эксперименты проводились за отраженными ударными волнами на высоковакуумной кинетической ударной трубе (УТ) в ОИВТ РАН. Труба с внутренним диаметром 108 мм и общей длиной 8 м изготовлена из нержавеющей стали. Камеры низкого и высокого давления (КНД и КВД), длиной 6 и 2 метра соответственно, разделены между собой в зависимости от перепада давления алюминиевой (80мкм) или медной (170мкм) диафрагмой. Откачка КНД осуществлялась последовательно безмасляным форвакуумным и турбомолекулярными насосами до давления $5 \times 10^{-7}$ мбар. При этом натекание в УТ составляло $8 \times 10^{-7}$ мбар/мин. Экспериментальная установка и оборудование схематически представлено на рис. 1. Более подробное описание установки приведено в работе [16].

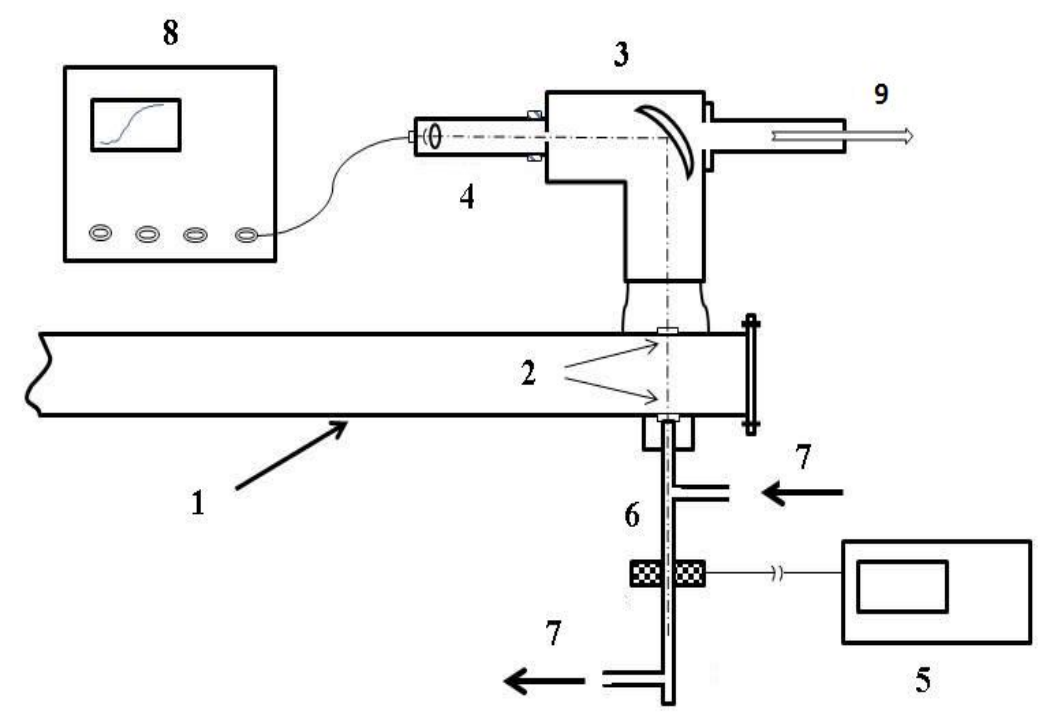

Рис. 1. Экспериментальная установка и схема измерений методом AРAC. 1 - ударная труба; 2 - окна из $\mathrm{MgF}_{2} ; 3$ - вакуумный монохроматор (BM); 4 - ФЭУ; 5 - твердотельный генератор; 6 - проточная кварцевая трубка (ПКТ); 7 - смесь $1 \% \mathrm{O}_{2}+\mathrm{He}$ при давлении $p=10$ мбар; 8 - осциллограф; 9 - форвакуумный насос для откачки ВМ

Исследуемые смеси составлялись на смесевой установке в сосуде из нержавеющей стали, также предварительно откачанном до давления $9 \times 10^{-7}$ мбар. Вследствие высокой чувствительности проводимых измерений к различным примесям, для создания смеси использовались только сверхчистые газовые компоненты: Ar чистоты 99.9999\%, $\mathrm{O}_{2}-99.999 \%$ (оба производства Линде Газ Рус) и СO - 99.99\% (производства SIAD).

Измерение концентрации атомарного кислорода осуществлялось с помощью метода атомно-резонансной абсорбционной спектроскопии (АРАC). Через проточную кварцевую 
трубку (ПКТ) диаметром 10 мм подавалась заранее составленная смесь $1 \% \mathrm{O}_{2}+$ Не при давлении 10 мбар. Давление в трубке измерялось вакуумметром и в случае необходимости корректировалось форвакуумным насосом. Далее в ПКТ с помощью источника микроволнового излучения создавался разряд. Источником являлся твердотельный генератор Sairem GMS-200 на частоте 2.45 ГГц с операционной мощностью 80 Вт. Под действием микроволнового разряда молекулы $\mathrm{O}_{2}$ диссоциировали, в результате создавая линейчатый спектр излучения атомарного кислорода, где наиболее сильная линия приходилась на длину волны 130.5 нм (рис. 2), что соответствовало основному триплетному переходу $\left({ }^{3} \mathrm{P}-{ }^{1} \mathrm{~S}_{1}\right)$. Излучение проходило через окна ударной трубы, вакуумный тракт и попадало на монохроматор Acton VM502, который, чтобы исключить поглощения УФ-излучения воздухом, откачивался форвакуумным насосом до давления $8 \times 10^{-3}$ мбар. В качестве детектора использовался ФЭУ-181 с областью чувствительности $112 \div 830$ нм. Эмиссионная линия вырезалась при параметрах входной и выходной щели монохроматора 30 мкм, что соответствовало спектральному разрешению в \pm 1.2 нм. Рабочее напряжение на ФЭУ варьировалось от 1400 до 1900 В. Подбор данных параметров проводился эмпирически для достижения наиболее качественного (порядка $40 \div 50$ ) соотношения сигнал/шум.

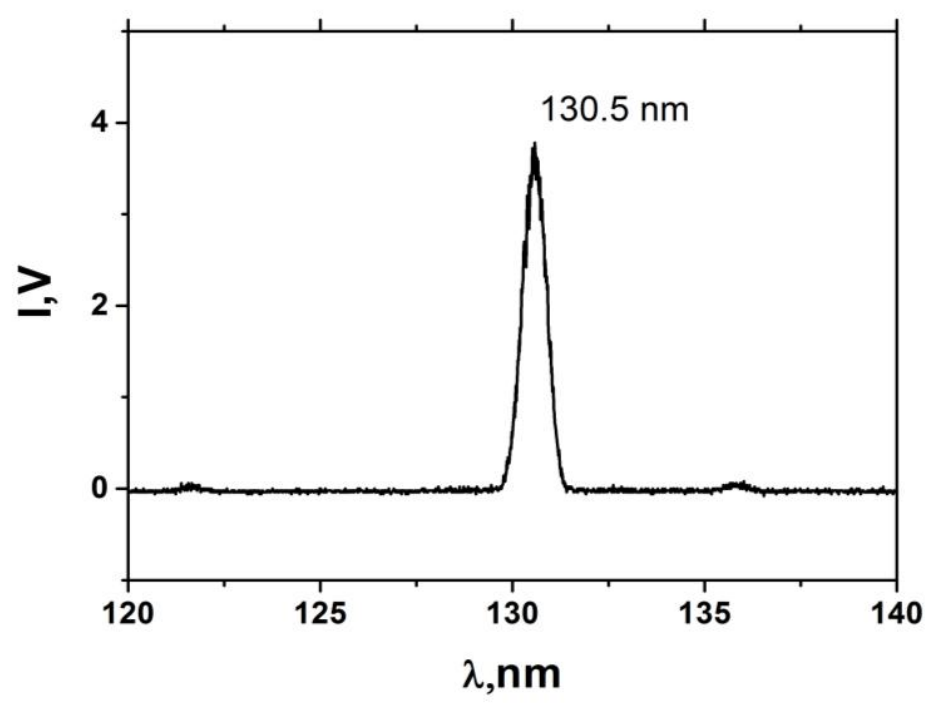

Рис. 2. Спектральная линия излучения микроволновой лампы в смеси $1 \% \mathrm{O}_{2}+\mathrm{He}$

Тестовые эксперименты, проведенные в чистом аргоне перед основной серией, подтвердили отсутствие какого-либо поглощения на данной длине волны.

\section{2. Калибровка резонансного поглощения атомарным кислородом}

Форма спектральной линии, полученная при излучении атомарного кислорода в лампе и зависимость ее поглощения от концентрации атомов кислорода в условиях данной экспериментальной схемы, известны лишь приблизительно. Поэтому, для возможности количественного перевода интенсивности поглощения атомами О в их концентрацию, необходимо проведение калибровочных экспериментов.

Серия калибровочных экспериментов проводилась на длине волны 130.5 нм в различных газовых смесях $\mathrm{N}_{2} \mathrm{O}+\mathrm{Ar}$ при температуре $2050 \pm 50 \mathrm{~K}$, давлении $2.35 \pm 0.20$ бар и концентрации $\mathrm{N}_{2} \mathrm{O}$ от 100 до $1 \mathrm{ppm}$. Данный диапазон температур и концентраций выбран подобно работам [17-18], когда реакция диссоциации $\mathrm{N}_{2} \mathrm{O}+\mathrm{Ar}=\mathrm{N}_{2}+\mathrm{O}+\mathrm{Ar}$ протекает достаточно быстро, а реакция рекомбинации $\mathrm{O}+\mathrm{O}+\mathrm{Ar}=\mathrm{O}_{2}+\mathrm{Ar}$ и другая вторичная кинетика ввиду малой концентрации практически отсутствует. Все это позволяет с достаточной точностью считать, что весь кислород за отраженной ударной волной находится в атомарной форме и его концентрация равна начальной концентрации $\mathrm{N}_{2} \mathrm{O}$. 
Представленная на рис. 3 типичная осциллограмма поглощения демонстрирует падение уровня сигнала излучения от микроволновой лампы, связанное с появлением атомов О при распаде оксида азота $\mathrm{N}_{2} \mathrm{O}$, и выход его на стационарный уровень. Измеряя этот уровень поглощения за отраженной ударной волной, и сопоставляя его с рассчитанным значением концентрации атомов О в текущем эксперименте, была построена калибровочная кривая (рис.4).

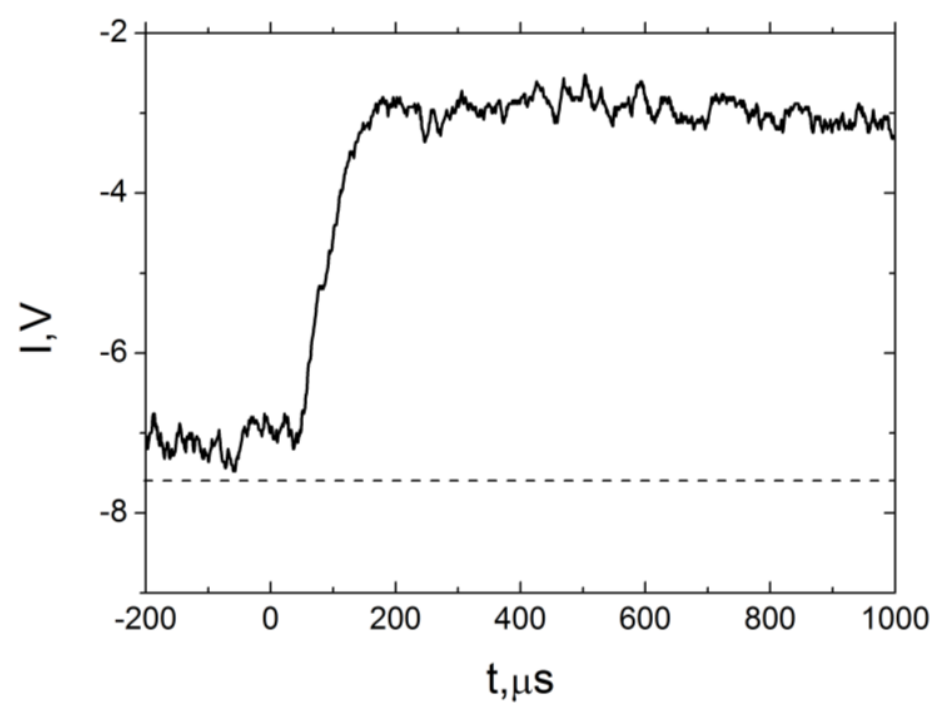

Рис. 3. Временной профиль поглощения на линии 130.5 нм в смеси $12.5 \mathrm{ppm}$ $\mathrm{N}_{2} \mathrm{O}+\mathrm{Ar} . T_{5}=2024 \mathrm{~K}, P_{5}=2.50$ атм. Пунктирная линия-величина сигнала при отсутствии поглощения

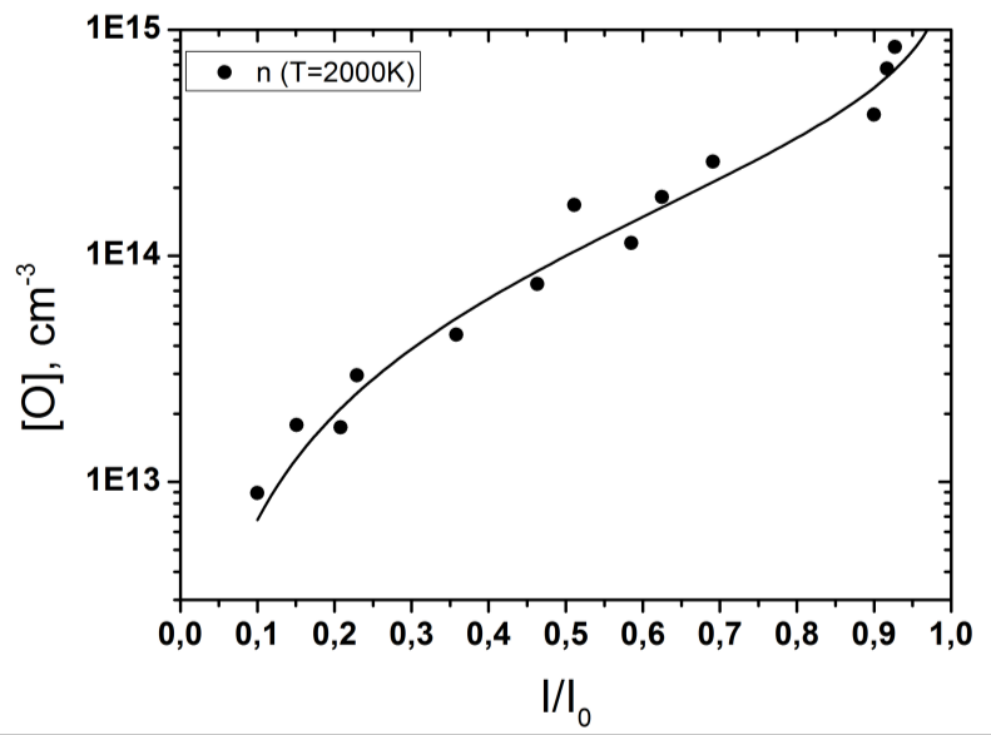

Рис. 4. Калибровочная кривая концентрации атомарного кислорода в смеси $\mathrm{N}_{2} \mathrm{O}+\mathrm{Ar}$ от интенсивности поглощения излучения на длине волны 130.5 нм

Из-за особенностей процессов уширения и сдвига поглощаемой линии, а также из-за неточно известной формы излучаемой в ПКТ линии [19], закон Бугера-Ламберта-Бэра не совсем точно отражает реальное соотношение между уровнем поглощения и концентрацией. Поэтому, в подобных случаях пользуются модификацией закона Бугера-Ламберта-Бэра, как это проделано в работах [19-20]

$$
\frac{I}{I_{0}}=1-\exp (\sigma L[\mathrm{O}])^{n}
$$


где $I / I_{0}$ - отношение интенсивности прошедшего через исследуемую смесь излучения к первоначальному; $\sigma$ - приведенное сечение поглощения; $L=10.8 \mathrm{~cm} \mathrm{-} \mathrm{оптическая} \mathrm{длина}$ (диаметр ударной трубы); [O] - концентрация атомарного кислорода в исследуемой смеси; $n$ - показатель степени. Коэффициенты $\sigma, n$ этого выражения определяются путем варьирования. Наилучшего согласия с экспериментальными данными удалось добиться при значении $n=0.7$ и $\sigma=5.5 \times 10^{-16} \mathrm{~cm}^{2}$. Далее, используя эту калибровочную зависимость, по уровню поглощения определялась текущая концентрация атомарного кислорода в различных реагирующих газовых смесях. Минимально регистрируемая величина концентрации составляла $8 \times 10^{12} \mathrm{~cm}^{-3}$, а погрешность измерений не превышала $30 \%$.

\section{3. Калибровка поглощения излучения молекулой СО на резонансной линии О}

Близкие к 130.5 нм линии и полосы других атомных и молекулярных компонентов, присутствующих в исследуемой смеси, из-за ограниченного спектрального разрешения монохроматора, могут вносить свой вклад в суммарное поглощение исходящего от источника излучения. Представленный в работе [21] спектр молекулы СО указывает на возможность ее поглощения в области 130.5 нм, поэтому на этой линии были проведены специальные эксперименты в смеси $\mathrm{CO}+\mathrm{Ar}$ для анализа количественной величины этого поглощения.

В этой серии экспериментов температура за отраженной ударной волной варьировалась от $1200 \mathrm{~K}$ до $1700 \mathrm{~K}$, а концентрация CO в Ar от $1 \%$ до $0.001 \%$. Эксперименты проводились при давлениях $2.5 \pm 0.1$ бар и $16 \pm 1$ бар. Пример типичной осциллограммы поглощения приведен на рис. 5.

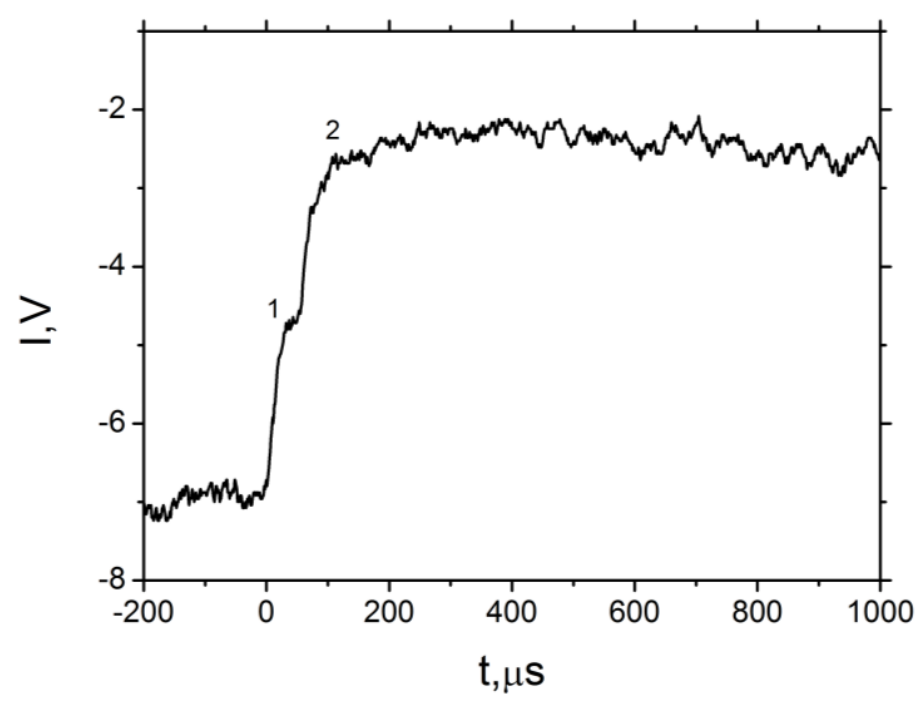

Рис. 5. Временной профиль поглощения на линии 130.5 нм в смеси $1000 \mathrm{ppm} \mathrm{CO}+\mathrm{Ar} . T_{5}=1496 \mathrm{~K}, P_{5}=16.798$ атм. $1-$ поглощение $\mathrm{CO}$ за ПУВ; 2 - поглощение СО за ОУВ

Поскольку выбранный температурный диапазон полностью исключает диссоциацию монооксида углерода [22], то наблюдаемые на рис. 5 резкие скачки уровня сигнала поглощения за падающей (область 1 рис. 5) и отраженной (область 2 рис. 5) ударной волной однозначно интерпретируются ростом давления и, соответственно, ростом абсолютной величины концентрации СО в соответствующих областях. Полученные результаты по паразитному поглощению молекулами СО на исследуемой длине волны учитывались при проведении основной серии экспериментов. 


\section{4. Поглощение излучения молекулой $\mathrm{O}_{2}$ на резонансной линии О}

Согласно данным работы [23] молекулярный кислород в области $130 \div 175$ нм имеет полосы поглощения, называемые континуумом Шумана-Рунге. Резонансная линия атомарного кислорода лежит на краю этой области, что может вызывать поглощение испускаемого излучения. Однако предварительные эксперименты, проведенные в смеси $0.1 \% \mathrm{O}_{2}+\mathrm{Ar}$ при температурах $1200 \div 1800 \mathrm{~K}$, показали, что поглощение молекулярным кислородом излучения на линии 130.5 нм по уровню не превышает шума. Нулевое поглощение также указывает на отсутствие значимой диссоциации молекулярного кислорода в этом диапазоне температур, что подтверждается большинством работ, например, [24], где диссоциация $\mathrm{O}_{2}$ заметна при температуре выше $3000 \mathrm{~K}$, поэтому в дальнейшем справедливо считалось, что молекулярный кислород не вносит вклад в суммарное поглощение.

\section{3. Результаты}

\section{1. Взаимодействие $\mathrm{CO}+\mathrm{O}_{2}$ в аргоне}

Взаимодействие молекулярного кислорода с оксидом углерода описывается с помощью следующей химической реакции:

$$
\mathrm{O}_{2}+\mathrm{CO}+\mathrm{Ar}=\mathrm{CO}_{2}+\mathrm{O}+\mathrm{Ar}
$$

Изучение взаимодействия $\mathrm{CO}+\mathrm{O}_{2}$ проводилось за отраженными ударными волнами в температурном диапазоне от 1200 до $2200 \mathrm{~K}$ при давлениях $2.55 \pm 0.20$ бар и $16.5 \pm 1.0$ бар. Первоначальный состав смеси в каждом эксперименте оставался неизменным и составлял $0.1 \% \mathrm{O}_{2}+0.1 \% \mathrm{CO}+\mathrm{Ar}$. Характерный пример полученной осциллограммы приведен на рис. 6. На основании предварительного анализа можно сделать вывод, что сигнал состоит из суммарного поглощения излучения молекулой $\mathrm{CO}$, связанного со скачками концентрации (область 1 и 2 на рис. 6) и поглощения атомарным кислородом, образовавшимся в ходе реакции (2) (область 3 на рис. 6).

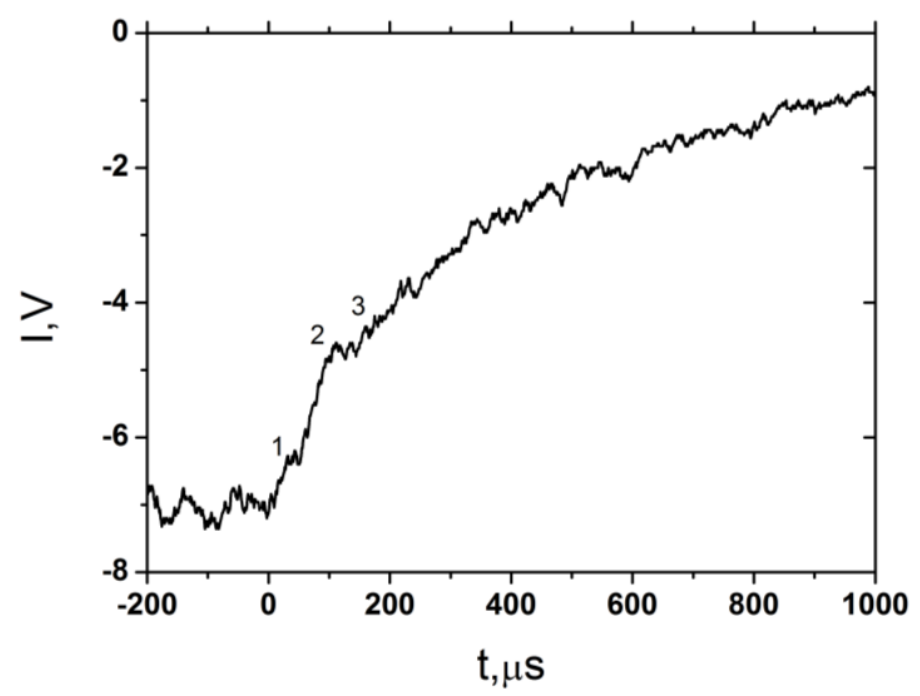

Рис. 6. Профиль поглощения в смеси $0.1 \% \mathrm{O}_{2}+0.1 \% \mathrm{CO}+\mathrm{Ar} T_{5}=1751 \mathrm{~K}$, $P_{5}=2.50$ атм. 1 - поглощение $\mathrm{CO}$ за ПУВ; 2 - поглощение $\mathrm{CO}$ за ОУВ; 3 - поглощение в результате протекания реакции $\mathrm{CO}_{2}+\mathrm{O}_{2}$

После скачка поглощения за ОУВ, перед дальнейшим монотонным изменением сигнала, видна временная задержка (участок кривой 2-3, рис.6). Хорошо известно, что процесс установления равновесия по колебательным степеням свободы в молекуле СО является относительно медленным. Поэтому, при ударно-волновом инициировании химической реакции 
(2), ее начальная стадия может протекать в отсутствии колебательного равновесия [25] (т.е. $\tau_{c} \approx \tau_{v}$, где $\tau_{c}$ и $\tau_{v}$ - характерные времена химической реакции и колебательной релаксации соответственно), что при недостаточном уровне колебательной энергии молекул реагентов ( $T>T_{v}$, где $T_{v}$ - колебательная температура) может существенно тормозить реакцию. Для проверки этого предположения выполнен замер временных задержек поглощения атомами кислорода и произведен расчет времени колебательного возбуждения двухатомных молекул СО при температурах (до $\sim 10^{4} \mathrm{~K}$ ) по механизму Ландау-Теллера [26]. Результаты определения времен колебательной релаксации СО представлены на рис. 7. Несмотря на существующий разброс экспериментальных точек, соответствие с теоретическим расчетом выглядит довольно убедительно. На основании этого можно полагать, что задержка сигнала поглощения (участок 2-3 на рис. 6) возникает именно в результате существенного замедления реакции (2) вследствие присутствующей на начальном этапе колебательной неравновесности.

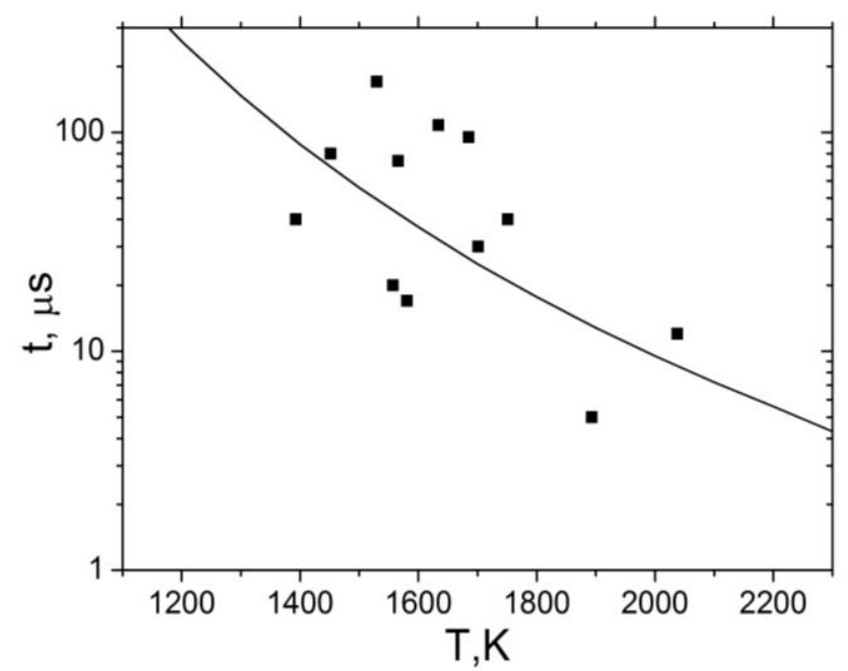

Рис. 7. Времена колебательной релаксации СО в зависимости от температуры газа за ОУВ. Сплошная линия - зависимость Ландау-Теллера. Квадратные точки - экспериментальные измерения

Основное кинетическое уравнение для реакции (2), где скорость реакции равна изменению во времени концентрации атомарного кислорода $(r=\mathrm{d}[\mathrm{O}] / \mathrm{d} t)$, можно записать в следующем виде:

$$
\frac{\mathrm{d}[\mathrm{O}]}{\mathrm{d} t}=k_{f}^{*}\left[\mathrm{O}_{2}\right][\mathrm{CO}][\mathrm{Ar}]-k_{r}^{*}\left[\mathrm{CO}_{2}\right][\mathrm{O}][\mathrm{Ar}]
$$

где $k_{f}^{*}$ - константа скорости прямой реакции; $k_{r}^{*}-$ константа скорости обратной реакции; $[\mathrm{N}]$ - концентрации соответствующих компонентов. При протекании химической реакции (2) атомы аргона несут функцию перераспределения энергии между взаимодействующими веществами. При этом их концентрация остается постоянной и на несколько порядков превышает концентрацию других компонентов реагирующей смеси. Это позволяет рассмотреть изучаемую реакцию как реакцию псевдо-второго порядка и перезаписать соотношение (3) в виде

$$
\frac{\mathrm{d}[\mathrm{O}]}{\mathrm{d} t}=k_{f}\left[\mathrm{O}_{2}\right][\mathrm{CO}]-k_{r}\left[\mathrm{CO}_{2}\right][\mathrm{O}]
$$

Все дальнейшие рассуждения будем вести, исходя именно из этого предположения. 


\section{2. Константа скорости прямой реакции}

В начальный момент времени, когда концентрации продуктов реакции (2) в исследуемой смеси пренебрежимо малы, константу скорости прямой реакции можно найти по тангенсу максимального угла наклона начального участка кинетической кривой (рис. 8, угол между прямой и осью абсцисс) по выражению

$$
k_{f}=\frac{\mathrm{d}[\mathrm{O}] / \mathrm{d} t}{[\mathrm{CO}]\left[\mathrm{O}_{2}\right]}
$$

Кинетическую кривую для каждого эксперимента (рис. 8) получали из осциллограмм поглощения с помощью полученной ранее калибровочной зависимости (1). Причем из суммарного сигнала поглощения, перед нормировкой по калибровочной кривой, вычиталось поглощение молекул СО.

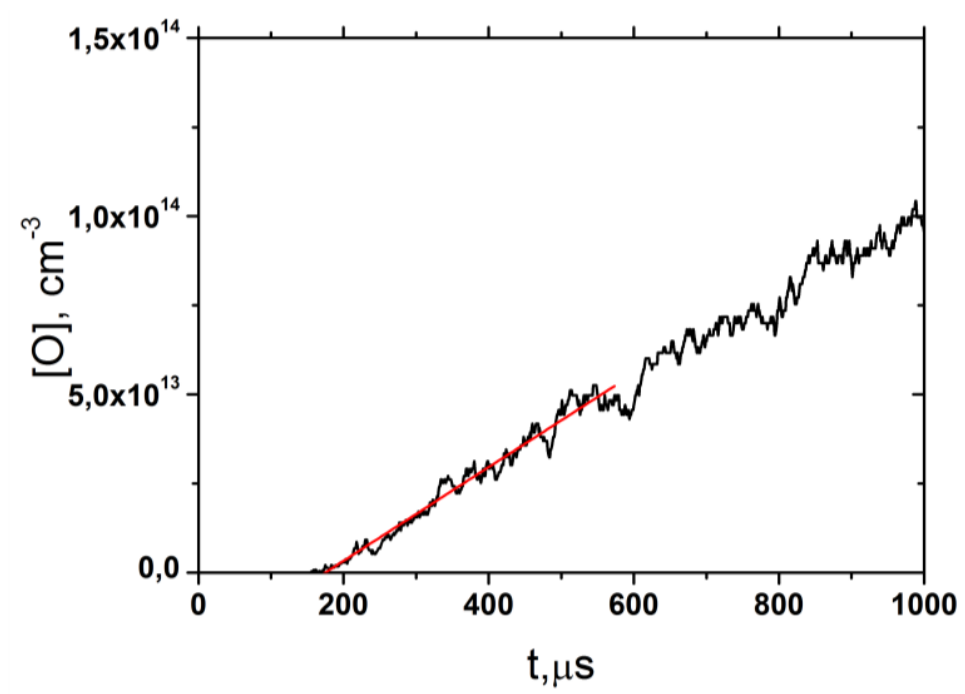

Рис. 8. Кинетическая кривая атомарного кислорода в смеси $0.1 \% \mathrm{O}_{2}+0.1 \% \mathrm{CO}+\mathrm{Ar} T_{5}=1751 \mathrm{~K}, P_{5}=2.50 \mathrm{aTM}$

Результаты, полученные из наших экспериментальных данных, вместе с результатами других авторов представлены на рис. 9.

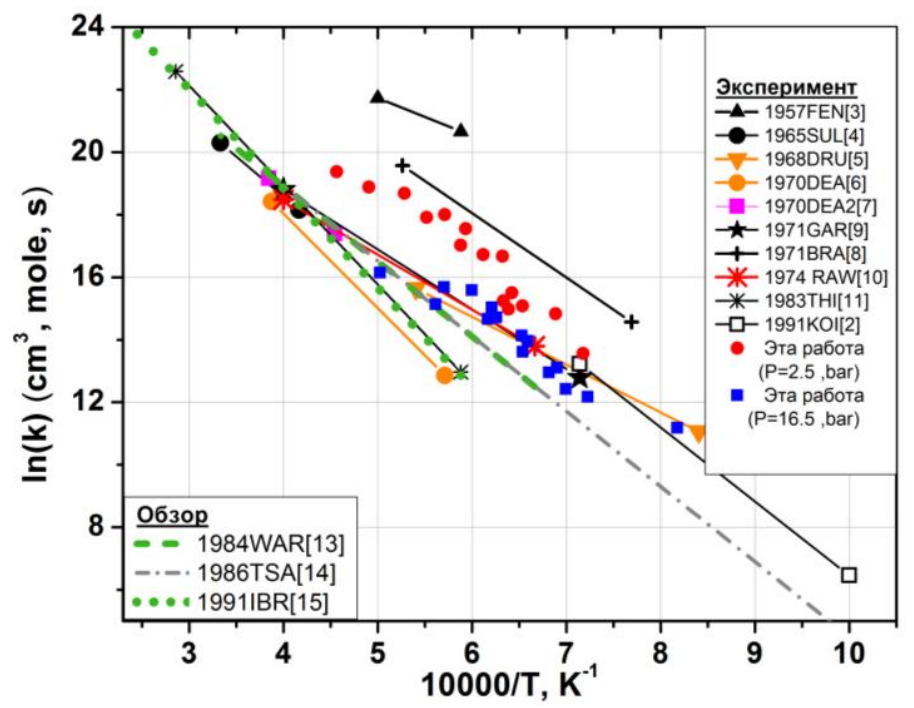

Рис. 9. Константа скорости прямой реакции (2) в сравнении с ранее полученными данными других авторов 
Значения константы скорости прямой реакции «расслаиваются» в зависимости от давления газа. Поэтому, энергия активации $E$ и предэкспоненциальный множитель $A$ определены каждые для своей величины давления за отраженной ударной волной. При этом константа скорости прямой реакции в аррениусовской форме $k_{f}=A T^{m} \exp (-E / R T)$, где $R-$ универсальная газовая постоянная, $T$ - температура, $m$ - показатель степени, имеет вид

$$
\begin{gathered}
k_{f H}=3.17 \times 10^{11} \exp \left(-\frac{167 \mathrm{~kJ}}{R T}\right), \mathrm{cm}^{3} \cdot \text { моль }^{-1} \cdot \mathrm{c}^{-1}-\text { при высоком давлении }(P=16.5 \text { бар }),(6) \\
k_{f L}=1.08 \times 10^{13} \exp \left(-\frac{167 \mathrm{~kJ}}{R T}\right), \mathrm{cm}^{3} \cdot \text { моль }^{-1} \cdot \mathrm{c}^{-1}-\text { при низком давления }(P=2.5 \text { бар) }
\end{gathered}
$$

\section{4. Обсуждение}

\section{1. Влияние давления на величину константы скорости прямой реакции}

Полученная экспериментальная зависимость константы скорости реакции от величины давления не может быть объяснена в рамках формальной кинетики. Уравнение Аррениуса вообще в явном виде не содержит функциональной зависимости от давления. Для объяснения наблюдаемого эффекта были использованы подходы теории активированного комплекса (TAK).

Основное положение теории активированного комплекса базируется на идее образования переходного состояния, представляющего собой активированный комплекс (АК) из взаимодействующих молекул, находящийся в равновесии с исходными веществами

$$
\mathrm{AB}+\mathrm{C} \stackrel{k_{1}, k_{-1}}{\longrightarrow} \mathrm{ABC}^{*} \stackrel{k_{2}}{\longrightarrow} \mathrm{A}+\mathrm{BC}
$$

При этом нами было высказано предположение, что каждый активированный комплекс переходит в продукты только в том случае, если за время его жизни не успевает произойти столкновительного взаимодействия с другими молекулами, т.е. рассматривается только спонтанный переход. С физической точки зрения такое предположение имеет простую интерпретацию: если после возбуждения переходного состояния, активированный комплекс за свое характерное время жизни успевает провзаимодействовать с другими молекулами, процесс идет в сторону первоначальных реагентов - АК тушится. Если же взаимодействие не произошло, АК спонтанно переходит в продукты. Очевидно, что процесс учитывает влияние давления, так как взаимодействие молекул с АК напрямую зависит от частоты газокинетических соударений, которые в свою очередь пропорциональны величине концентрации.

Теперь получим зависимость константы скорости от давления, опираясь на сделанные предположения. Аналогично методам формальной кинетики составим кинетическую схему процесса

$$
\begin{aligned}
& \mathrm{CO}+\mathrm{O}_{2} \stackrel{k_{1}}{\longrightarrow}\left(\mathrm{CO}: \mathrm{O}_{2}\right)^{*}, \\
& \left(\mathrm{CO}: \mathrm{O}_{2}\right)^{*}+\mathrm{M} \stackrel{k_{-1}}{\longrightarrow} \mathrm{CO}+\mathrm{O}_{2}, \\
& \left(\mathrm{CO}: \mathrm{O}_{2}\right)^{*}+\mathrm{M} \stackrel{k_{2}}{\longrightarrow} \mathrm{CO}_{2}+\mathrm{O},
\end{aligned}
$$

где $\left(\mathrm{CO}: \mathrm{O}_{2}\right)^{*}$ - есть некий АК с определенным временем жизни; $k_{1}, k_{-1}, k_{2}$ - константы скорости реакции (9), (10) и (11) соответственно.

Для схемы (9-11) составим основное кинетическое уравнение. Из соотношения (11) и (10) получим 


$$
\begin{gathered}
\frac{\mathrm{d}[\mathrm{O}]}{\mathrm{d} t}=k_{2}\left[\left(\mathrm{CO}: \mathrm{O}_{2}\right)^{*}\right] \\
\frac{\mathrm{d}\left[\mathrm{CO}: \mathrm{O}_{2}\right]}{\mathrm{d} t}=k_{1}[\mathrm{CO}]\left[\mathrm{O}_{2}\right]-k_{-1}\left[\left(\mathrm{CO}: \mathrm{O}_{2}\right)^{*}\right][\mathrm{M}]-k_{2}\left[\left(\mathrm{CO}: \mathrm{O}_{2}\right)^{*}\right]
\end{gathered}
$$

Используя условие квазистационарности концентрации $\mathrm{AK} \mathrm{d}\left[\mathrm{CO}: \mathrm{O}_{2}\right]^{*} / \mathrm{d} t=0$, как в схеме Линдемана или теории РРКМ, получим следующее выражение:

$$
\left[\left(\mathrm{CO}: \mathrm{O}_{2}\right)^{*}\right]=\frac{k_{1}[\mathrm{CO}]\left[\mathrm{O}_{2}\right]}{k_{-1}[\mathrm{M}]+k_{2}}
$$

Подставив (14) в (12), окончательно получим кинетическое уравнение на основе ТАК для кинетической схемы (9-11)

$$
\frac{\mathrm{d}[\mathrm{O}]}{\mathrm{d} t}=\frac{k_{2} k_{1}[\mathrm{CO}]\left[\mathrm{O}_{2}\right]}{k_{-1}[\mathrm{M}]+k_{2}}
$$

Сопоставив это выражение с кинетическим уравнением (5) $\mathrm{d}[\mathrm{O}] / \mathrm{d} t=k_{f}\left[\mathrm{O}_{2}\right][\mathrm{CO}]$, полученным ранее при рассмотрении реакции (2) методом формальной кинетики и решив систему (5), (15), получим связь феноменологической константы скорости реакции $\mathrm{CO}+\mathrm{O}_{2}$ с константами скорости, рассчитанными на основе ТАК

$$
k_{f}=\frac{k_{2} k_{1}}{k_{-1}[\mathrm{M}]+k_{2}}
$$

Как видно из выражения (16), феноменологическая константа скорости, полученная на основе экспериментальных расчетов, является некоторой эффективной константой, связывающей определенным соотношением константы скорости образования и распада АК. Из этого соотношения также наглядно видно влияние общей концентрации вещества на величину константы скорости, что и наблюдалось в наших экспериментах. Причем уменьшение концентрации увеличивает скорость реакции. В предельном случае, когда $k_{-1}[\mathrm{M}] \ll k_{2}, k_{f} \rightarrow k_{1}$ и ТАК дает тот же результат, что и формальная кинетика.

Далее проведем расчеты отдельных величин, входящих в выражение (15). Как уже утверждалось $k_{f}$ - константа, которая определена непосредственно из проведенного нами эксперимента. Посчитав частоту газокинетических соударений $V$, можно определить константу $k_{-1}$, которая связана с частотой соотношением $V=k_{2}[\mathrm{M}] / N_{\mathrm{A}}$, где $N_{\mathrm{A}}$ - число Авогадро. Константу скорости $k_{1}$ можно найти, зная энергию активации и свойства АК, а также рассчитав его сумму по состояниям, непосредственно из квантово-механических расчетов ТАК, [27]. Из-за отсутствия однозначно определенных свойств АК и сложности их расчета константа $k_{1}$ являлась в нашем случае варьируемой величиной, на которую наложены ряд ограничений по абсолютному значению и значению энергии активации. Так как реакции (10) и (11) являются безбарьерными, энергия активации $k_{1}$ не должна отличаться от величины, полученной экспериментально. За начальное приближение $k_{1}$ взято значение экспериментальной константы скорости при низком давлении $k_{f}$. Так как $k_{f}<k_{1}$ и стремится к $k_{1}$ при уменьшении концентрации, величину $k_{1}$ увеличивали до тех пор, пока метод последовательных итераций не давал хорошего согласия с экспериментом. В итоге, из соотношения (15) для каждого эксперимента была определена константа скорости $k_{2}$ (рис. 10). Таким образом, значения всех констант, рассчитанных по ТАК, были получены. Результаты расчета представлены в таблице 2 в аррениусовской форме $k=A \exp (-E / R T)$.

Интересно отметить, что обратная величина константы скорости $k_{2}, t=1 / k_{2}$ и есть время жизни активированного комплекса. При этом приближенно $t=(5 \pm 3) \times 10^{-10}$ с. Изна- 
чально в модели ТАК вводилось предположение, что переходное состояние имеет время жизни порядка частоты колебаний $\left(\sim 10^{-12} \mathrm{c}\right)$. Однако, дальнейшее развитие теории РРКМ предполагало возможность существования АК с временами жизни $\sim 10^{-10-9} \mathrm{c}$.

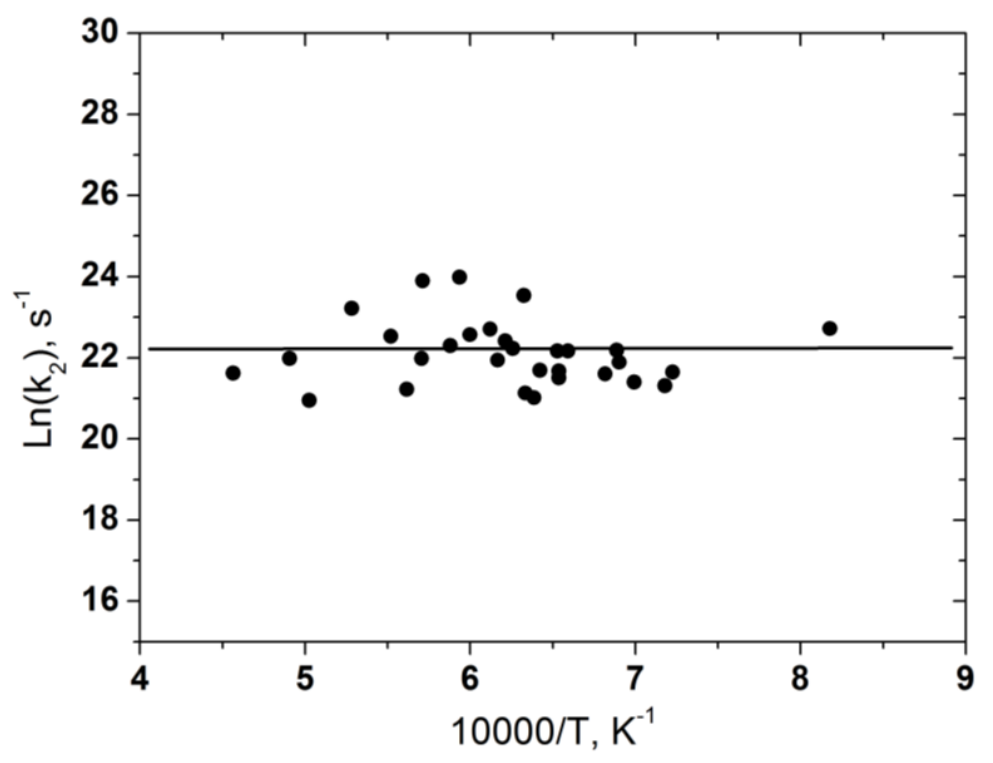

Рис. 10. Константа скорости $k_{2}$, Прямая линия - значение константы, полученное методом наименьших квадратов (МНК)

Таблищу 2

Значения констант скоростей реакции $\mathrm{CO}+\mathrm{O}_{2}$, вычисленных на основе ТАК

\begin{tabular}{|c|c|c|}
\hline \multirow{2}{*}{ Реакция } & \multicolumn{2}{|c|}{$k=A \exp (-E / R T)$} \\
\cline { 2 - 3 } & $A, \mathrm{~cm}^{3} /$ моль ${ }^{*} \mathrm{c}$ & $E$, кДж/моль \\
\hline $\mathrm{CO}+\mathrm{O}_{2}=\left(\mathrm{CO}: \mathrm{O}_{2}\right)^{*}$ & $8.3 \mathrm{e} 13$ & 167 \\
\hline$\left(\mathrm{CO}: \mathrm{O}_{2}\right)^{*}+\mathrm{Ar}=\mathrm{CO}+\mathrm{O}_{2}$ & $3.6 \mathrm{e} 14$ & 0 \\
\hline$\left(\mathrm{CO}: \mathrm{O}_{2}\right)^{*}+\mathrm{Ar}=\mathrm{CO}_{2}+\mathrm{O}$ & $1.3 \mathrm{e} 9(1 / \mathrm{c})$ & 0 \\
\hline
\end{tabular}

Впервые долгоживущие комплексы объяснены и обоснованы Хиншельвудом [27], внесшим поправки в схему активации молекул Линдемана - Крамерса. Он предположил, что превращение активной молекулы происходит после сосредоточения энергии, равной или превышающей энергию активации, в определенной части молекулы. Вероятность сосредоточения энергии на разрываемой связи зависит от числа колебательных и возможно вращательных степеней свободы, а также ее прочности. Поэтому на такое перераспределение может требоваться сотни и даже тысячи колебаний.

Для проверки теоретических результатов, были проведены расчеты с использованием полученных констант с помощью пакета программ «Chemkin» при высоком и низком давлении. Итогом расчетов стало сравнение экспериментальных и смоделированных профилей концентрации атомарного кислорода (рис. $11, a, \sigma$ ), а также оценка давления, при котором становится заметным «расслоение» константы скорости (значение температуры практически не вносит вклад в «расслоение» константы скорости, так как реакции (10), (11) являются безбарьерными). Согласно модели, влияние общего давления газа на константу скорости (2) начинает проявляться при давлении выше или порядка 0.1 бар. Однако, для экспериментального детектирования эффекта «расслоения» требуются эксперименты на порядок и более отличающиеся по общей концентрации газа. Возможно поэтому, в работах [5, 8-10], этот эффект 
не был зарегистрирован. Лишь при давлении порядка 10 бар и более зависимость константы скорости от величины давления проявляется существенно. Как видно из рис. 11, линейный рост концентрации кислорода, наблюдаемый в расчетах, близок по форме к профилям экспериментальных кривых.
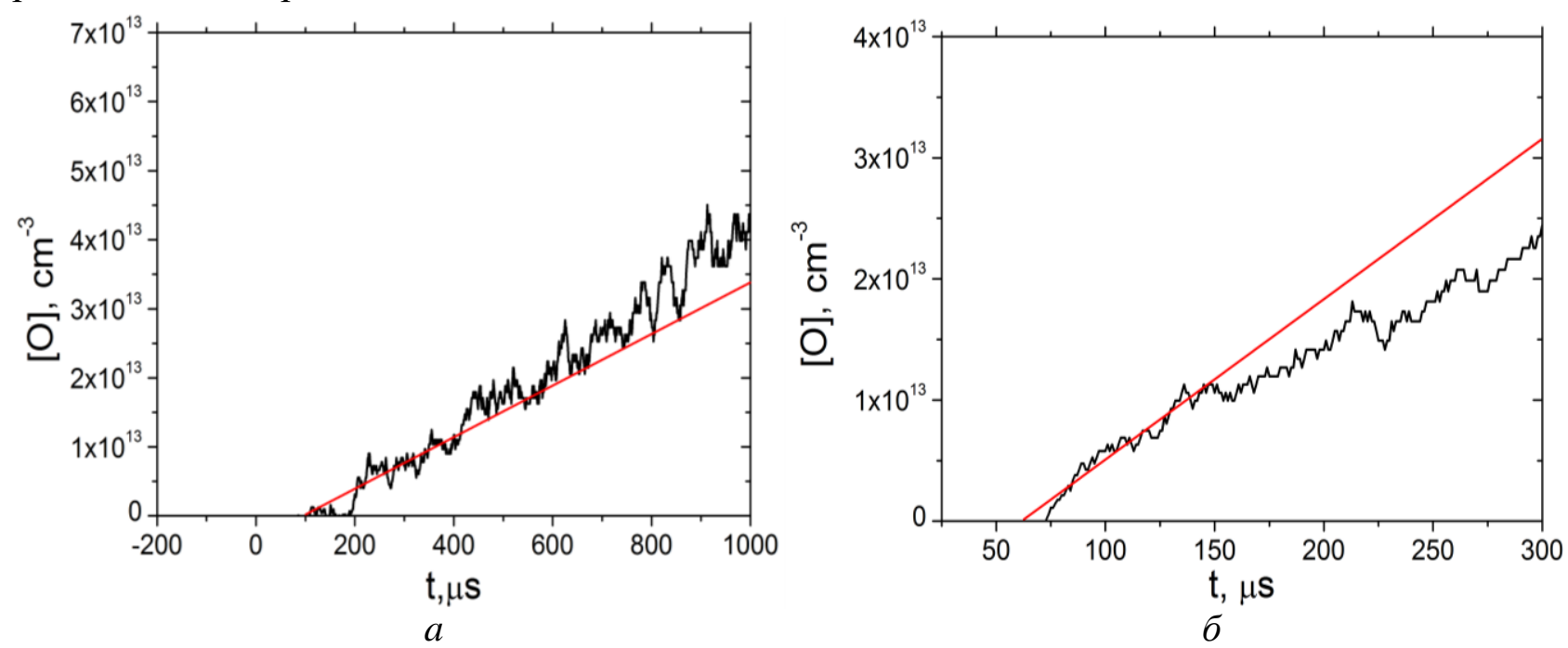

Рис. 11. Сравнение экспериментальных и теоретически полученных профилей концентрации атомарного кислорода в смеси $0.1 \% \mathrm{O}_{2}+0.1 \% \mathrm{CO}+\mathrm{Ar}$. Красная прямая - теоретический расчет; черная кривая - эксперимент; a) $T_{5}=1634 \mathrm{~K}, P_{5}=2.60$ бар; б) $T_{5}=1530 \mathrm{~K}, P_{5}=16.90$ бар

Это подтверждает, что на изучаемом начальном временном отрезке влияние вторичных реакций мало, и кинетика реакции (2) может быть описана в рамках ТАК, базируясь на кинетической схеме (9-12). В начальные моменты времени согласие модели с экспериментом по росту концентрации атомарного кислорода как при низком, так и при высоком давлении выглядит убедительным. Погрешность модели не превышает $30 \%$. Расхождение расчета и эксперимента при высоком давлении на более поздней стадии реакции объясняется насыщением сигнала поглощения. Если при низком давлении суммарное поглощение медленно росло на протяжении всего времени наблюдения и доходило не более чем до $70 \div 80 \%$, то при высоком, за время $200 \div 300$ мкс, сигнал достигал уровня насыщения в $90 \div 100 \%$. Дальнейший рост концентрации кислорода, который естественно имел место, становилось невозможно зарегистрировать экспериментально. При обработке полученных осциллограмм с учетом вычитания из осциллограмм стационарного уровня поглощения $\mathrm{CO}$, сигнал оставался на некотором заниженном уровне, а значит, после применения калибровочной зависимости, на заниженном уровне оставалась и концентрация кислорода. Таким образом, как только уровень поглощения достигает 90\% и более, информативность сигнала быстро падает, корректное преобразование интенсивности в концентрацию становится невозможным. В итоге при высоком давлении модель предсказывает более вероятный результат развития реакции на поздней стадии, чем наши экспериментальные данные.

\section{5. Заключение}

Техника атомной резонансной абсорбционной спектроскопии была успешно применена для получения временных профилей концентрации атомарного кислорода, образовавшегося в результате взаимодействия окиси углерода с молекулярным кислородом в смеси $0.1 \% \mathrm{O}_{2}+0.1 \% \mathrm{CO}+\mathrm{Ar}$ за отраженными ударными волнами. Минимально зарегистрированная величина концентрации атомов О составляла порядка $8 \times 10^{12} \mathrm{~cm}^{-3}$. Константа скорости прямой реакции $\mathrm{O}_{2}+\mathrm{CO}+\mathrm{Ar}=\mathrm{CO}_{2}+\mathrm{O}+\mathrm{Ar}(2)$ получена для широкого диапазона температур $(1200 \div 2200 \mathrm{~K})$ при высоком и низком давлении. Экспериментально выявлено, что величина константы скорости прямой реакции (2) падает при повышении давления газа. Полученные результаты проанализированы в рамках теории активированного комплекса. В результате, 
рассчитаны константы скоростей образования, тушения и спонтанного распада активированного комплекса $\left(\mathrm{CO}: \mathrm{O}_{2}\right)^{*}$. Определенное время жизни комплекса $\left(\mathrm{O}_{2}: \mathrm{CO}\right)$ составляло $0.5 \pm 0.3$ нс. Проведенное с использованием полученных констант моделирование показывает хорошее соответствие результатов расчета и эксперимента.

\section{Благодарности}

Работа поддержана грантом Российского Фонда Фундаментальных Исследований, грант РФФИ № 17-08-01303.

\section{Литература}

1. Fenimore C.P., Jones G.W. The water-catalyzed oxidation of carbon monoxide by oxygen at high temperature// J. Phys. Chem. 1957. V.61. P. 651.

2. Koike T. Shock tube studies of the $\mathrm{H}_{2}-\mathrm{O}_{2}-\mathrm{CO}$ reaction by atomic resonance absorption spectroscopy// Bull. Chem. Soc. Jpn. 1991. V. 64. P. 1726.

3. Fenimore C.P., Jones G.W. The water-catalyzed oxidation of carbon monoxide by oxygen at high temperature// J. Phys. Chem. 1957. V.61. P. 651.

4. Sulzmann K.G.P., Myers B.F., Bartle E.R. CO oxidation. I. Induction period preceding $\mathrm{CO}_{2}$ formation in shock-heated CO-O 2 -Ar mixtures// J. Chem. Phys. 1965. V.42. P. 3969.

5. Drummond, L.J. Shock-initiated exothermic reactions. IV. The oxidation of carbon monoxide// Aust. J. Chem. 1968. V.21. P. 2631.

6. Dean A.M., Kistiakowsky G.B. Oxidation of carbon monoxide/methane mixtures in shock waves// J. Chem. Phys. 1970. V. 54. P. 1718.

7. Dean A.M., Kistiakowsky G.B. Oxidation of carbon monoxide by oxygen in shock waves// J. Chem. Phys 1970. V.53. P. 830.

8. Gardiner W.C. Jr., McFarland M., Morinaga K., Takeyama T., Walker B.F. Initiation rate for shock heated hydrogen-oxygen-carbon monoxide-argon mixtures as determined by $\mathrm{OH}$ induction time measurements// J. Phys. Chem. 1971. V.75. P. 1504.

9. Brabbs T.A., Belles F.E., Brokaw R.S. Shock-Tube Measurements of Specific Reaction Rates in the Branched-Chain $\mathrm{H}_{2}-\mathrm{CO}-\mathrm{O}_{2}$ System// Symp. Int. Combust. Proc. 1971. V.13. P. 129.

10. Rawlins W.T., Gardiner W.C. Rate Constant for $\mathrm{CO}+\mathrm{O}_{2} \rightarrow \mathrm{CO}_{2}+\mathrm{O}$ from 1500 to 2500 K. A Reevaluation of Induction Times in the Shock-Initiated Combustion of Hydrogen-Oxygen-Carbon Monoxide-Argon Mixtures// J. Phys. Chem. 1974. V.78. P. 497.

11. Thielen K., Roth P. Stosswellenuntersuchungen zum start der reaktion $\mathrm{CO}+\mathrm{O}_{2} / /$ Ber. Bunsenges. Phys. Chem. 1983. V.87. P. 920.

12. Chase, M.W., Jr., NIST-JANAF Themochemical Tables, Fourth Edition, J. Phys. Chem. Ref. Data, Monograph 9, 1998, 1-1951

13. Warnatz J. Rate coefficients in the $\mathrm{C} / \mathrm{H} / \mathrm{O}$ system. Chap. 5 in Combustion Chemistry (ed. Gardiner W.C. Jr.) Springer-Verlag, NY, 1984.

14. Tsang W., Hampson R.F. Chemical kinetic data base for combustion chemistry. Part I. Methane and related compounds// J. Phys. Chem. Ref. Data. 1986. V.15. P. 1087.

15. Ибрагимова Л.Б. Рекомендуемые константы скорости реакции $\mathrm{CO}+\mathrm{O}_{2} \leftrightarrow \mathrm{CO}_{2}+\mathrm{O} / /$ Химическая физика. 1991. том 10, № 3. с. 307.

16. Дракон А.В., Емельянов А.В., Еремин А.В., Яценко П.И. Исследование диссоциации трифторметана в широком диапазоне температур и давлений с использованием метода молекулярнорезонансной абсорбционной спектроскопии// ТВТ. 2017. Т.55 № 2. С. 245.

17. Dove J.E., Nip W.S., Teitelbaum H. The vibrational relaxation and pyrolysis of shock heated nitrous oxide// Symp. (Int.) cornbust. 1975. V.15. Pp. 903-916. 
18. Sdherland J.W., Klemtn R.B. Rate constants for the thermal dissociation of $\mathrm{N}_{2} \mathrm{O}$ and the $\mathrm{O}(3 \mathrm{P})+\mathrm{N}_{2} \mathrm{O}$ reaction// J. Phys. Chem. A. 1997. V. 101. Pp. 1104-1116.

19. Naudet V., Abid S. and Paillard C.E. A High Temperature Chemical Kinetics Study of the $\mathrm{O}_{2}$ Dissociation and the O Atoms Recombination by ARAS // J. Chem. Phys. 1999. V. 96. Pp. 1123-1145.

20. Catoire L., Woiki D., Roth P. A Shock Tube Study of the Reaction of H Atoms with $\mathrm{SiCl}_{4} / /$ Int. J. Chem. Kinet. 1997, V. 29. Pp. 469-472.

21. Myer J.A.,Samson J.A.R.// J. Chem. Phys., 1970. V.52. P. 266.

22. Mick H-J., Burmeister M., Roth P. Atomic resonance absorption spectroscopy measurements on hightemperature CO dissociation kinetics// AIAA J. 1993. V.31. P. 671.

23. Watanabe K., Inn E.C.Y., Zelikoff M. // J. Chem. Phys. 1953. V.21. P. 1026.

24. Naudet V., Abid S., Paillard C.S. A high temperature chemical kinetics study of the $\mathrm{O}_{2}$ dissociation and the O atoms recombination by ARAS// J. Chem. Phys. 1999. V. 96. P. 1123.

25. Полак Л.С. Неравновесная химическая кинетика и ее применение. М.: Наука, 1979.

26. Ландау Л.Д., Теллер Е. Собрание трудов. М.: Наука, 1969.

27. Кубасов А.А. Химическая кинетика и катализ. Часть 2. Теоретические основы химической кинетики// Москва, 2005.

Статья поступила в редакцию 29 ноября 2017 г. 